\title{
Calcium Affects on Vascular Endpoints (CAVE) Retrospective Cohort Trial
}

\author{
Rajib K. Bhattacharya ${ }^{1 *}$, Vaishali B. Patel ${ }^{1}$, James L. Vacek ${ }^{2}$ \\ ${ }^{1}$ Division of Endocrinology, Metabolism \& Genetics, The University of Kansas Medical Center, Kansas City, USA \\ ${ }^{2}$ Mid-America Cardiology, The University of Kansas Medical Center, Kansas City, USA \\ Email: ${ }^{*}$ rbhattacharya@kumc.edu
}

Received September 5, 2012; revised October 8, 2012; accepted November 12, 2012

\begin{abstract}
Purpose: The goal of this study was to examine the association of calcium supplements with coronary artery disease and survival. Our initial hypothesis was that when adjusted for risk factors, calcium supplements would not affect overall survival outcomes or coronary artery disease prevalence in a community population followed by a large cardiovascular practice. Methods Data was retrospectively collected for 5 years and 8 months (1/1/2004 to 10/8/2009) from the electronic medical record at a large cardiovascular practice at a university. Only patients $\geq$ aged 50 were included in our analysis. Results: The baseline characteristics of the 8060 study subjects. For the entire study population the mean age was $65+/-10$ years, $71 \%$ were women. On multivariable logistic regression analysis, calcium supplementation was not an independent predictor of all-cause death, nor was calcium supplementation an independent predictor of the diagnosis of coronary artery disease. Survival analysis showed no survival differences for patients on calcium supplements ( $p=$ NS). Hazard ratios using the Cox proportional hazards model were calculated, showing that calcium supplementation was not an independent predictor of reduced survival. Conclusion: This study suggests that there was no association between calcium supplementation and coronary artery disease as well as overall survival in a large retrospective cohort of patients greater than 50 with high rates of significant cardiovascular risk factors.
\end{abstract}

Keywords: Calcium Supplements; Heart Attacks; Coronary Artery Disease; Cardiovascular Risk Factors

\section{Introduction}

Calcium supplements are widely used in the United States and other developed nations, traditionally to avoid osteopenia and its complications, but now often given along with vitamin D supplementation for maintenance of optimum health for other physiologic processes, including the cardiovascular system [1-4]. A secondary analysis of a randomized placebo controlled trial of calcium supplements versus placebo in post-menopausal women for 5 years showed a statistically significant increase in composite end points in a calcium group vs. no supplementation [5]. There have been recent studies and meta-analyses that have sparked a national debate on the cardiovascular safety of calcium supplementation [6]. The majority of these studies did not include major cardiovascular endpoints in their primary objectives [7-11]. Many of the studies did not have patient level data regarding cardiovascular outcomes [12-16].

The goal of this study was to examine the association of calcium supplements with coronary artery disease and survival. Our initial hypothesis was that when adjusted

"Corresponding author. for risk factors, calcium supplements would not affect overall survival outcomes or coronary artery disease prevalence in a community population followed by a large cardiovascular practice at a Midwestern university academic medical center. These outcomes were adjusted for demographics, cardiovascular risk factors and potentially confounding medications.

\section{Methods}

Data was retrospectively electronically collected for 5 years and 8 months (1/1/2004 to $10 / 8 / 2009)$ from the electronic medical record of Mid America Cardiology, a large cardiovascular practice at the University of Kansas Hospital and Medical Center in Kansas City, Kansas. Patients $\geq$ age 50 were included in our analysis. Our laboratory used the Beckman DXC 800 for the basic metabolic profile including calcium levels. The laboratory used DiaSorin $^{\circledR}$ (Stillwater, MN) chemiluminescence immunoassay method to measure total serum vitamin D (both 25 hydroxy D2 and D3 forms of vitamin D). Diagnoses were derived from the patient problem list in the patients' electronic medical record based on International Classification of Diseases, 9th Revision 
(ICD-9) codes. Death was determined from the Social Security Death Index. The study population consisted of 8060 patients. The dosage for the calcium supplement was assumed to be $500 \mathrm{mg}$ if the dosage was not documented.

\section{Statistical Analysis}

This data was entered and formatted in Microsoft Excel 2007. The data was then imported into SAS 9.1.3 for statistical analysis and modeling. Descriptive statistics, univariate analysis (unpaired $t$ tests for continuous variables, chi square analysis for categorical variables), multivariable logistic regression for odds ratios (OR) and confidence intervals (CI), survival analysis using the Kaplan Meier method and log rank testing, and Cox proportional hazard modeling were performed. Survival was calculated using the interval between thedate of sample collection and the date of death or the end of the study period. A 0.05 level of significance was used throughout. Multiple iterations of the logistic and hazards routines were performed to assess various models and variable combinations.

\section{Results}

The baseline characteristics of the 8060 study subjects are in Table 1, categorized by calcium supplement use. For the entire study population the mean age was $65+/-$ 10 years, $71 \%$ were women. A significantly higher proportion of the individuals in the supplement cohort were

Table 1. Baseline characteristics.

\begin{tabular}{|c|c|c|c|}
\hline \multirow[t]{2}{*}{ Variables } & \multicolumn{2}{|c|}{ Calcium supplementation } & \multirow[t]{2}{*}{$\mathrm{p}$ value } \\
\hline & Yes & No & \\
\hline $\mathrm{N}(\%)$ & $2878(36 \%)$ & $5182(64 \%)$ & \\
\hline Age (years) & $67+/-11$ & $64+/-10$ & $<0.0001$ \\
\hline Women & $2402(83 \%)$ & $3329(64 \%)$ & $<0.0001$ \\
\hline Body mass index $\left(\mathrm{kg} / \mathrm{m}^{2}\right)$ & $29+/-7$ & $30+/-8$ & $<0.0001$ \\
\hline Coronary artery disease $(>70 \%$ stenosis $\geq 1$ coronary artery) & $475(17 \%)$ & $599(12 \%)$ & $<0.0001$ \\
\hline Cardiomyopathy & $159(6 \%)$ & $176(3 \%)$ & $<0.0001$ \\
\hline Hypertension & $1428(50 \%)$ & $1767(34 \%)$ & $<0.0001$ \\
\hline Diabetes mellitus & $505(18 \%)$ & $924(18 \%)$ & NS \\
\hline Valvular heart disease & $290(10 \%)$ & $305(6 \%)$ & $<0.0001$ \\
\hline Atrial fibrillation & $265(9 \%)$ & $303(6 \%)$ & $<0.0001$ \\
\hline Ejection fraction $(\%)$ & $57+/-10$ & $57+/-11$ & NS \\
\hline Aspirin use & $1473(51 \%)$ & $1469(28 \%)$ & $<0.0001$ \\
\hline Angiotensin-converting enzyme inhibitor use & $1084(38 \%)$ & $1300(25 \%)$ & $<0.0001$ \\
\hline Statin use & $1582(55 \%)$ & $1698(33 \%)$ & $<0.0001$ \\
\hline Creatinine (mg/dl) & $1.19+/-1.01$ & $1.70+/-3.54$ & $<0.0001$ \\
\hline Total cholesterol (mg/dl) & $170+/-45$ & $166+/-48$ & 0.02 \\
\hline High-density lipoproteincholesterol (mg/dl) & $52+/-18$ & $46+/-15$ & $<0.0001$ \\
\hline Low density lipoprotein cholesterol (mg/dl) & $95+/-36$ & $94+/-39$ & NS \\
\hline Vitamin D level (ng/ml) & $28+/-13$ & $23+/-14$ & $<0.0001$ \\
\hline Vitamin D supplement & $1407(49 \%)$ & $989(19 \%)$ & $<0.0001$ \\
\hline Death & $94(3 \%)$ & $192(4 \%)$ & NS \\
\hline Triglycerides (mg/dl) & $127+/-94$ & $136+/-121$ & NS \\
\hline Any vitamin & $1881(65 \%)$ & $1350(26 \%)$ & $<0.0001$ \\
\hline Calcium level (mg/dl) & $9.30+/-0.60$ & $9.16+/-0.57$ & $<0.0001$ \\
\hline
\end{tabular}


Table 2. Logistic regression for death as dependent variable.

\begin{tabular}{cccc}
\hline Predictor & OR & CI & p \\
\hline Calcium supplement & 0.862 & $0.650-1.117$ & $<0.0001$ \\
Coronary artery disease & 2.071 & $1.545-2.777$ & $<0.0001$ \\
Age & 1.025 & $1.013-1.036$ & 0.004 \\
Diabetes mellitus & 1.523 & $1.148-2.020$ & $<0.0001$ \\
Cardiomyopathy & 2.821 & $1.982-4.014$ & 0.019 \\
Hypertension & 1.391 & $1.057-1.832$ & $<0.0001$ \\
Female & 0.435 & $0.336-0.564$ & \\
\hline
\end{tabular}

Table 3. Proportional hazards analysis for death.

\begin{tabular}{ccccc}
\hline Variable & Hazard ratio & 95\% Hazard ratio confidence limits & $\mathrm{p}$ \\
\hline Female gender & 0.442 & 0.344 & 0.567 & $<0.0001$ \\
Calcium supplement & 0.762 & 0.587 & 0.989 & 0.0412 \\
Age & 1.023 & 1.012 & 1.035 & $<0.0001$ \\
Cardiomyopathy & 2.455 & 1.777 & 3.392 & $<0.0001$ \\
Hypertension & 1.475 & 1.127 & 1.930 & 0.0046 \\
Coronary artery disease & 2.058 & 1.551 & 2.732 & $<0.0001$ \\
Diabetes & 1.540 & 1.175 & 2.018 & 0.0017 \\
\hline
\end{tabular}

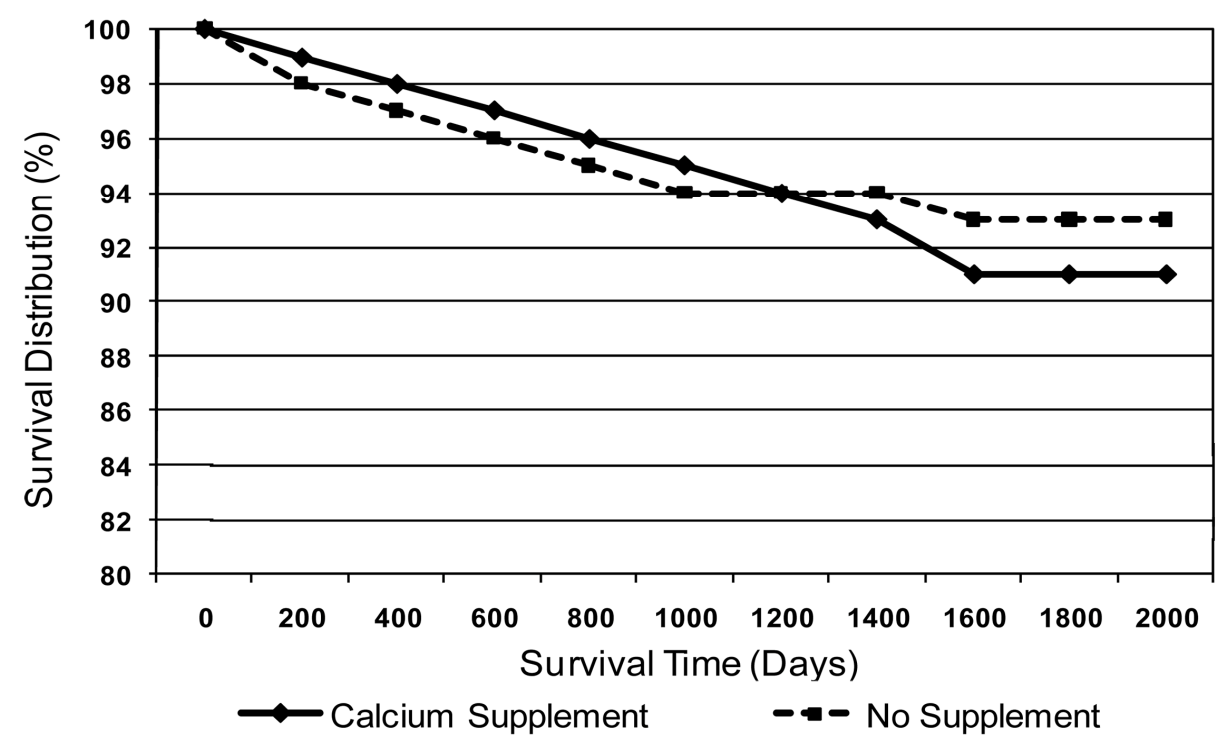

Figure 1. Survival analysis between individuals on calcium supplement vs no supplement.

on statins, aspirin, angiotensin-converting enzyme inhibittors and vitamin D supplementation.

On multivariable logistic regression analysis, calcium supplementation was not an independent predictor of allcause death (Table 2), nor was calcium supplementation an independent predictor of the diagnosis of coronary artery disease. Survival analysis showed no survival differences for patients on calcium supplements $(p=N S)$ (Figure 1). Hazard ratios using the Cox proportional hazards model were calculated, showing that calcium supplementation was not an independent predictor of reduced survival (Table 3). Hazard function analysis with additional predictive variables was then performed and again no overall significant impact of calcium supplementation on survival was evident.

\section{Conclusions}

Our study suggests that calcium supplements were not 
associated with reduction in survival or the diagnosis of coronary artery disease in this study population. We were able to document and adjust for important cardiovascular risk factors, clinical variables and preventative medications that influence survival. This study population was likely at higher risk for cardiovascular outcomes due to its derivation from a cardiology practice setting. We confirmed our initial hypothesis that calcium supplements did not increase the risk of certain cardiovascular diseases or overall mortality.

Our study should be considered in the context of previously published investigations. A secondary analysis of a randomized placebo controlled trial of calcium supplements versus placebo in post-menopausal women for 5 years showed a statistically significant increase in MI. The composite end point was higher in calcium group with a relative risk of 1.66 . When the events were attempted to be adjudicated with a national database, statistical significance was no longer found [5]. The baseline characteristics of the participants in this study were not adjusted for cardiovascular risk nor was there adequate documentation for concomitant cardiovascular medications.

The Women's Health Initiative trial, a randomized controlled study did not show any concerning cardiovascular risk from calcium supplements. This study revealed that calcium/vitamin D supplementation had no effect on the risk for CHD or stroke in generally healthy postmenopausal women throughout the 7-year duration of this randomized trial $[8,7]$.

A meta-analysis of 11 studies which included 12,000 participants showed that allocation to calcium supplements $(>500 \mathrm{mg} / \mathrm{day}$ for more than a year) was associated with increased risk of MI. Calcium supplementation was associated with $30 \%$ increase in incidence of MI, and smaller non-significant increase in the risks of CVA and death [6]. Few of the trials had CV events as the primary end points and data on CV events were not gathered in a standardized manner.

A prospective cohort study of 34,486 postmenopausal Iowa women 55 - 69 years old and without a history of ischemic heart disease was analyzed to investigate whether greater intakes of calcium, vitamin D, or milk products may influence ischemic heart disease mortality. This report did not show a clear trend for calcium supplementation influencing cardiovascular events [17]. In addition an excellent 5-year randomized, placebo controlled trial of calcium carbonate developed for the primary purpose to evaluate major cardiovascular endpoint and mortality just concluded. The calcium group did not show increase risk of cardiovascular events and mortality when compared to placebo, rather the calcium supplements showed a decrease in hospitalizations and mortality [18].

Our retrospective trial attempted to document impor- tant cardiovascular risk factors and other confounding variables which may have impacted the association of calcium supplementation with coronary artery disease and survival. Our study did have limitations. Calcium supplementation intake was self-reported. If the patient was noncompliant or simply stopped the medication it would not have been documented. In addition the calcium supplementation group had higher usage of aspirin, angiotensin converting enzyme inhibitors and statins, which we adjusted for by logistic regression. This was not a randomized prospective controlled trial, therefore we could not equalize the different subgroups. However the calcium supplementation group did have higher rates of cardiovascular risk factors and worse overall cardiac status at baseline (Table 1).

\section{Summary}

This study suggests that there was no association between calcium supplementation and coronary artery disease as well as overall survival in a large retrospective cohort of patients greater than 50 with high rates of significant cardiovascular risk factors. To our knowledge this is one of the largest retrospective studies calcium supplementation studies that have been adjusted for cardiovascular risk factors, disease states, ejection fraction and medications.

\section{REFERENCES}

[1] R. P. Heaney, "The Calcium Controversy: Finding a Middle Ground between the Extremes," Public Health Reports, Vol. 104, 1989, pp. 36-46.

[2] C. C. Johnston Jr., J. Z. Miller, C. W. Slemenda, et al., "Calcium Supplementation and Increases in Bone Mineral Density in Children," The New England Journal of Medicine, Vol. 327, No. 2, 1992, pp. 82-87. doi:10.1056/NEJM199207093270204

[3] P. J. Elders, P. Lips, J. C. Netelenbos, et al., "Long-Term Effect of Calcium Supplementation on Bone Loss in Perimenopausal Women," Journal of Bone and Mineral Research: The Official Journal of the American Society for Bone and Mineral Research, Vol. 9, No. 7, 1994, pp. 963-970.

[4] B. M. Tang, G. D. Eslick, C. Nowson, et al., "Use of Calcium or Calcium in Combination with Vitamin D Supplementation to Prevent Fractures and Bone Loss in People Aged 50 Years and Older: A Meta-Analysis," The Lancet, Vol. 370, No. 9588, 2007, pp. 657-666. doi:10.1016/S0140-6736(07)61342-7

[5] M. J. Bolland, P. A. Barber, R. N. Doughty, et al., "Vascular Events in Healthy Older Women Receiving Calcium Supplementation: Randomised Controlled Trial," BMJ, Vol. 336, No. 7638, 2008, pp. 262-266. doi:10.1136/bmj.39440.525752.BE

[6] M. J. Bolland, A. Avenell, J. A. Baron, et al., "Effect of Calcium Supplements on Risk of Myocardial Infarction 
and Cardiovascular Events: Meta-Analysis," BMJ, Vol. 341, 2010, p. c3691. doi:10.1136/bmj.c3691

[7] J. Hsia, G. Heiss, H. Ren, et al., "Calcium/Vitamin D Supplementation and Cardiovascular Events," Circulation, Vol. 115, No. 7, 2007, pp. 846-854. doi:10.1161/CIRCULATIONAHA.106.673491

[8] J. E. Manson, M. A. Allison, J. J. Carr, et al., "Calcium/ Vitamin D Supplementation and Coronary Artery Calcification in the Women's Health Initiative," Menopause, Vol. 17, No. 4, 2010, pp. 683-691.

[9] I. R. Reid, R. W. Ames, M. C. Evans, et al., "Effect of Calcium Supplementation on Bone Loss in Postmenopausal Women," The New England Journal of Medicine, Vol. 328, No. 7, 1993, pp. 460-464. doi:10.1056/NEJM199302183280702

[10] A. M. Grant, A. Avenell, M. K. Campbell, et al., "Oral Vitamin $\mathrm{D}_{3}$ and Calcium for Secondary Prevention of Low-Trauma Fractures in Elderly People (Randomised Evaluation of Calcium or Vitamin D, RECORD): A Randomised Placebo-Controlled Trial," The Lancet, Vol. 365, No. 9471, 2005, pp. 1621-1628. doi:10.1016/S0140-6736(05)63013-9

[11] I. R. Reid, B. Mason, A. Horne, et al., "Randomized Controlled Trial of Calcium in Healthy Older Women," The American Journal of Medicine, Vol. 119, No. 9, 2006, pp. 777-785. doi:10.1016/j.amjmed.2006.02.038

[12] B. Dawson-Hughes, G. E. Dallal, E. A. Krall, et al., "A Controlled Trial of the Effect of Calcium Supplementation on Bone Density in Postmenopausal Women," The New England Journal of Medicine, Vol. 323, No. 13, 1990, pp. 878-883. doi:10.1056/NEJM199009273231305

[13] B. L. Riggs, W. M. O'Fallon, J. Muhs, et al., "Long-Term
Effects of Calcium Supplementation on Serum Parathyroid Hormone Level, Bone Turnover, and Bone Loss in Elderly Women," Journal of Bone and Mineral Research: The Official Journal of the American Society for Bone and Mineral Research, Vol. 13, No. 2, 1998, pp. 168-174.

[14] R. L. Prince, A. Devine, S. S. Dhaliwal and I. M. Dick, "Effects of Calcium Supplementation on Clinical Fracture and Bone Structure: Results of a 5-Year, Double-Blind, Placebo-Controlled Trial in Elderly Women," Archives of Internal Medicine, Vol. 166, No. 8, 2006, pp. 869-875.

[15] S. Bonnick, S. Broy, F. Kaiser, et al., "Treatment with Alendronate Plus Calcium, Alendronate Alone, or Calcium Alone for Postmenopausal Low Bone Mineral Density," Current Medical Research and Opinion, Vol. 23, No. 6, 2007, pp. 1341-1349. doi:10.1185/030079907X188035

[16] J. M. Lappe, D. Travers-Gustafson, K. M. Davies, et al., "Vitamin D and Calcium Supplementation Reduces Cancer Risk: Results of a Randomized Trial," The American Journal of Clinical Nutrition, Vol. 85, No. 6, 2007, pp. 1586-1591.

[17] R. M. Bostick, L. H. Kushi, Y. Wu, et al., "Relation of Calcium, Vitamin D, and Dairy Food Intake to Ischemic Heart Disease Mortality among Postmenopausal Women," American Journal of Epidemiology, Vol. 149, No. 2, 1999, pp. 151-161. doi:10.1093/oxfordjournals.aje.a009781

[18] J. R. Lewis, J. Calver, K. Zhu, et al., "Calcium Supplementation and the Risks of Atherosclerotic Vascular Disease in Older Women: Results of a 5-Year RCT and a 4.5-Year Follow-Up," Journal of Bone and Mineral Research: The Official Journal of the American Society for Bone and Mineral Research, Vol. 26, No. 1, 2011, pp. 3541. 